\title{
CERTAIN INTEGRALS INVOLVING ALEPH FUNCTION AND WRIGHT'S GENERALIZED HYPERGEOMETRIC FUNCTION
}

\author{
D.L. Suthar, H. Habenom, H. Tadesse
}

ABstract. The aim of this paper is to establish certain integrals involving product of the Aleph function with exponential function and Fox-Wright's Generalized Hypergeometric Function. Being unified and general in nature, these integrals yield a number of known and new results as special cases. For the sake of illustration, four corollaries are also recorded here as special case of our main results.

2010 Mathematics Subject Classification: 33C45, 33C60.

Keywords: Aleph function, Fox-Wright's Generalized Hypergeometric function H- Function, I-function, Mellin-Barnes type contour integral.

\section{Introduction AND Preliminaries}

Throughout this paper, let $\mathbb{C}, \mathbb{R}, \mathbb{Z}^{-}$, and $\mathbb{N}$ be sets of complex numbers, real numbers, and positive integers respectively. Also $\mathbb{N}_{0}:=\{0\} \cup \mathbb{N}$.

The Aleph function, which is a general higher transcendental function and was introduced by $\ddot{S}$ udland et al. [9, 10], is defined in terms of the Mellin- Barnes type integrals as following manner (see, e.g., $[7,8]$ )

$$
\begin{aligned}
\aleph[z] & =\aleph_{p_{i}, q_{i}, \tau_{i} ; r}^{m, n}\left[z \mid \begin{array}{c}
\left(a_{j}, \alpha_{j}\right)_{1, n}, \ldots,\left[\tau_{i}\left(a_{j i}, \alpha_{j i}\right)\right]_{n+1, p_{i} ; r} \\
\left(b_{j}, \beta_{j}\right)_{1, m}, \ldots,\left[\tau_{i}\left(b_{j i}, \beta_{j i}\right)\right]_{m+1, q_{i} ; r}
\end{array}\right] \\
& =\frac{1}{2 \pi i} \int_{L} \Omega_{p_{i}, q_{i}, \tau_{i} ; r}^{m, n}(\xi) z^{-\xi} d \xi,
\end{aligned}
$$

where $z \in \mathbb{C}-\{0\}, i=\sqrt{-1}$ and

$$
\Omega_{p_{i}, q_{i}, \tau_{i} ; r}^{m, n}(\xi)=\frac{\Pi_{j=1}^{m} \Gamma\left(b_{j}+\beta_{j} \xi\right) \Pi_{j=1}^{n} \Gamma\left(1-a_{j}-\alpha_{j} \xi\right)}{\sum_{i=1}^{r} \tau_{i} \Pi_{j=m+1}^{q_{i}} \Gamma\left(1-b_{j i}-\beta_{j i} \xi\right) \Pi_{j=n+1}^{p_{i}} \Gamma\left(a_{j i}+\alpha_{j i} \xi\right)},
$$

The integration path $L=L_{i \gamma \infty}(\gamma \in \mathbb{R})$ ranging from $\gamma-i \infty$ to $\gamma-i \infty$; is a contour of the Mellin-Barnes type, which separates the poles of $\Gamma\left(1-a_{j}-\alpha_{j} \xi\right),(j=$ 
$1, \ldots, n)$ from $\Gamma\left(b_{j}+\beta_{j} \xi\right),(j=1, \ldots, m)$. The empty product in $(2)$ is interpreted as unity. The parameters $p_{i}, q_{i} \in \mathbb{N}_{0}$ with $0 \leq n \leq p_{i}, 1 \leq m \leq q_{i}$ ,$\tau_{i}>0(i=1, \ldots, r)$ and $\alpha_{j}, \beta_{j}, \alpha_{j i}, \beta_{j i}>0$ whereas $a_{j}, b_{j}, a_{j i}, b_{j i} \in \mathbb{C}$. The existence of the $\aleph$-function defined on (1) depends on the following conditions.

$$
\varphi_{l}>0,|\arg (z)|<\frac{\pi}{2} \varphi_{l}, l=1, \ldots, r
$$

and

$$
\varphi_{l} \geq 0,|\arg (z)|<\frac{\pi}{2} \varphi_{l}, l=1, \ldots, r \text { and } \Re\left(\zeta_{l}\right)+1<0
$$

where

$$
\varphi_{l}=\sum_{j=1}^{n} \alpha_{j}+\sum_{j=1}^{m} \beta_{j}-\tau_{l}\left(\sum_{j=n+1}^{p_{l}} \alpha_{j l}+\sum_{j=m+1}^{q_{l}} \beta_{j l}\right)
$$

and

$$
\zeta_{l}=\sum_{j=1}^{m} b_{j}-\sum_{j=1}^{n} a_{j}+\tau_{l}\left(\sum_{j=m+1}^{q_{l}} b_{j l}-\sum_{j=n+1}^{p_{l}} a_{j l}\right)+\frac{1}{2}\left(p_{l}-q_{l}\right), l=1, \ldots, r
$$

Remark 1: On setting $\tau_{i}=1(i=1, \ldots, r)$ in (1), yields the I-function due to Saxena [6], defined in following manner:

$$
\begin{aligned}
I_{p_{i}, q_{i} ; r}^{m, n}[z]=\aleph_{p_{i}, q_{i}, 1 ; r}^{m, n}[z] & =\aleph_{p_{i}, q_{i}, 1 ; r}^{m, n}\left[z \mid \begin{array}{c}
\left(a_{j}, \alpha_{j}\right)_{1, n}, \ldots,\left[\left(a_{j i}, \alpha_{j i}\right)\right]_{n+1, p_{i}} \\
\left(b_{j}, \beta_{j}\right)_{1, m}, \ldots,\left[\left(b_{j i}, \beta_{j i}\right)\right]_{m+1, q_{i}}
\end{array}\right] \\
& =\frac{1}{2 \pi i} \int_{L} \Omega_{p_{i}, q_{i}, 1 ; r}^{m, n}(\xi) z^{-\xi} d \xi .
\end{aligned}
$$

where the kernel $\Omega_{p_{i}, q_{i}, 1 ; r}^{m, n}(\xi)$ is given in (2). The existence conditions for the integral in (7) are the same as given in (3)-(6) with $\tau_{i}=1(i=1, \ldots, r)$.

Remark 2: If we set $r=1$, then (7) reduces to the familiar H-function [3]

$$
H_{p, q}^{m, n}[z]=\aleph_{p_{i}, q_{i}, 1 ; 1}^{m, n}[z]=\aleph_{p_{i}, q_{i, 1 ; 1}}^{m, n}\left[z \mid \begin{array}{c}
\left(a_{j}, \alpha_{j}\right) \\
\left(b_{j}, \beta_{j}\right)
\end{array}\right]=\frac{1}{2 \pi i} \int_{L} \Omega_{p_{i}, q_{i, 1 ; 1}}^{m, n}(\xi) z^{-\xi} d \xi .
$$

are the kernel $\Omega_{p_{i}, q_{i, 1 ; 1}}^{m, n}(\xi)$ can be obtained from (2).

For our purpose, we recall the Wright's Generalized hypergeometric function ${ }_{p} \psi_{q}$ given by Wright [12] defined as:

$$
{ }_{p} \psi_{q}\left[\begin{array}{c}
\left(e_{1}, \nu_{1}\right), \ldots,\left(e_{p}, \nu_{p}\right) ; \\
\left(f_{1}, \varepsilon_{1}\right), \ldots,\left(f_{p}, \varepsilon_{p}\right) ;
\end{array}\right]={ }_{p} \psi_{q}\left[\begin{array}{c}
\left(e_{j}, \nu_{j}\right)_{1, p} ; \\
\left(f_{j}, \varepsilon_{j}\right)_{1, q} ;
\end{array}\right]=\sum_{k=0}^{\infty} \frac{\prod_{j=1}^{p}\left(e_{j}+\nu_{j} k\right)}{\prod_{j=1}^{q}\left(f_{j}+\varepsilon_{j} k\right)} \frac{x^{k}}{k !} .
$$


D.L. Suthar, H. Habenom, H. Tadesse - Certain Integrals Involving ...

where $\nu_{j}$ and $\varepsilon_{j}(i=1, \ldots, p ; j=1, \ldots, q)$ are real and positive, and $1+\sum_{j=1}^{q} \varepsilon_{j}-$ $\sum_{j=1}^{p} \nu_{j}>0$.

To establish the integrals, we have also used the following result due to Rainville [4]:

$$
\sum_{n=0}^{\infty} \sum_{k=0}^{\infty} A(k, n)=\sum_{n=0}^{\infty} \sum_{k=0}^{n} A(k, n-k) .
$$

\section{MAin Results}

In this section, we have evaluated three integrals involving product of the Aleph function and Wright's Generalized Hypergeometric function.

\section{First Integral}

$$
\begin{gathered}
I_{1} \equiv \int_{0}^{t} x^{\rho-1}(t-x)^{\delta-1} e^{-x z}{ }_{p} \psi_{q}\left(a x^{\varsigma}(t-x)^{\eta}\right) \\
\times \aleph_{p_{i}, q_{i}, \tau_{i}: r}^{m, n}\left[y x^{\mu}(t-x)^{v} \mid \begin{array}{c}
\left(a_{j}, \alpha_{j}\right)_{1, n}, \ldots,\left[\tau_{i}\left(a_{j i}, \alpha_{j i}\right)\right]_{n+1, p_{i} ; r} \\
\left(b_{j}, \beta_{j}\right)_{1, m}, \ldots,\left[\tau_{i}\left(b_{j i}, \beta_{j i}\right)\right]_{m+1, q_{i} ; r}
\end{array}\right] d x \\
=e^{-z t} t^{\rho+\delta-1} \sum_{u=0}^{\infty} \sum_{k=0}^{u} \Lambda(k) \frac{z^{(u-k)}}{(u-k) !} t^{u+(\varsigma+\eta-1) k} \\
\times \aleph_{p_{i}+2, q_{i}+1, \tau_{i}: r}^{m, n+2}\left[y t^{\left.(\mu+v) \mid \begin{array}{c}
(1-\rho-\varsigma k, \mu),(1-\delta-(\eta-1) k-u, v),\left(a_{j}, \alpha_{j}\right)_{1, n}, \ldots,\left[\tau_{i}\left(a_{j i}, \alpha_{j i}\right)\right]_{n+1, p_{i} ; r} \\
(1-\rho-\delta-u-(\varsigma+\eta-1) k, \mu+v),\left(b_{j}, \beta_{j}\right)_{1, m}, \ldots,\left[\tau_{i}\left(b_{j i}, \beta_{j i}\right)\right]_{m+1, q_{i} ; r}
\end{array}\right] .}\right.
\end{gathered}
$$

where

$$
\Lambda(k)=\frac{\prod_{j=1}^{p}\left(e_{j}+\nu_{j} k\right) a^{k}}{\prod_{j=1}^{q}\left(f_{j}+\varepsilon_{j} k\right) k !}
$$

provided

1. $\mu \geq 0, v \geq 0$ (not both zero simultaneously) such that $v-\mu>0$,

2. $\zeta$ and $\eta$ are non-negative integers such that $\zeta+\eta \geq 1$,

3. $A_{i}>0, B_{i}<0 ;|\arg (y)|<\frac{1}{2} A_{i} \pi, \forall i \in 1, \ldots, r ;$ where

$$
A_{i}=\sum_{j=1}^{n} \alpha_{j}+\sum_{j=1}^{m} \beta_{j}-\tau_{i}\left(\sum_{j=n+1}^{p_{i}} \alpha_{j i}+\sum_{j=m+1}^{q_{i}} \beta_{j i}\right)
$$


D.L. Suthar, H. Habenom, H. Tadesse - Certain Integrals Involving ...

$$
B_{i}=\frac{1}{2}\left(p_{i}-q_{i}\right)+\left(\sum_{j=1}^{m} b_{j}-\sum_{j=1}^{n} a_{j}\right)+\tau_{i}\left(\sum_{j=m+1}^{q_{i}} b_{j i}-\sum_{j=n+1}^{p_{i}} a_{j i}\right)
$$

4. $\Re(\rho)+\mu \min _{1 \leq j \leq m}\left[\Re\left(b_{j} / \beta_{j}\right)\right]>0, \quad \Re(\delta)+v \min _{1 \leq j \leq m}\left[\Re\left(b_{j} / \beta_{j}\right)\right]>0$.

Proof:

$$
\begin{gathered}
I_{1} \equiv e^{-z t} \int_{0}^{t} x^{\rho-1}(t-x)^{\delta-1} e^{(t-x) z_{p} \psi_{q}\left(a x^{\varsigma}(t-x)^{\eta}\right)} \\
\times \aleph_{p_{i}, q_{i}, \tau_{i}: r}^{m, n}\left[y x^{\mu}(t-x)^{v} \mid \begin{array}{c}
\left(a_{j}, \alpha_{j}\right)_{1, n}, \ldots,\left[\tau_{i}\left(a_{j i}, \alpha_{j i}\right)\right]_{n+1, p_{i} ; r} \\
\left(b_{j}, \beta_{j}\right)_{1, m}, \ldots,\left[\tau_{i}\left(b_{j i}, \beta_{j i}\right)\right]_{m+1, q_{i} ; r}
\end{array}\right] d x,
\end{gathered}
$$

Substituting the $e^{(t-x) z}$ by the infinite series $\sum_{u=0}^{\infty} \frac{(t-x)^{u} z^{u}}{u !}$ and rewriting the Aleph (※) function and Wright generalized hypergeometric function the with the help of (1) and (9) respectively, we obtain;

$$
\begin{gathered}
I_{1} \equiv e^{-z t} \int_{0}^{t} x^{\rho-1}(t-x)^{\delta-1} \sum_{u=0}^{\infty} \frac{(t-x)^{u} z^{u}}{u !} \sum_{k=0}^{\infty} \frac{\prod_{j=1}^{p}\left(e_{j}+\nu_{j} k\right)}{\prod_{j=1}^{q}\left(f_{j}+\varepsilon_{j} k\right)} \frac{a^{k} x^{\varsigma k}(t-x)^{\eta k}}{k !} \\
=e^{-z t} \int_{0}^{t} x^{\rho-1}(t-x)^{\delta-1} \sum_{u=0}^{\infty} \sum_{k=0}^{\infty} \frac{\prod_{j=1}^{p}\left(e_{j}+\nu_{j} k\right)}{\prod_{j=1}^{q}\left(f_{j}+\varepsilon_{j} k\right)} \frac{a^{k} z^{u} x^{\varsigma k}(t-x)^{\eta k+u}}{k ! u !} \\
\times \frac{1}{2 \pi i} \int_{L} \Omega_{p_{i}, q_{i}, \tau_{i}: r}^{m}(\xi) y^{-\xi} x^{-\mu \xi}(t-x)^{-v \xi} d \xi d x,
\end{gathered}
$$

By means of (8), the above result reduces to

$$
\begin{aligned}
=e^{-z t} \int_{0}^{t} x^{\rho-1}( & t-x)^{\delta-1} \sum_{u=0}^{\infty} \sum_{k=0}^{u} \frac{\prod_{j=1}^{p}\left(e_{j}+\nu_{j} k\right)}{\prod_{j=1}^{q}\left(f_{j}+\varepsilon_{j} k\right)} \frac{a^{k} x^{\varsigma k}(t-x)^{\eta k+u-k}}{k !} \frac{z^{(u-k)}}{(u-k) !} \\
& \times \frac{1}{2 \pi i} \int_{L} \Omega_{p_{i}, q_{i}, \tau_{i}: r}^{m, n}(\xi) y^{-\xi} x^{-\mu \xi}(t-x)^{-v \xi} d \xi d x,
\end{aligned}
$$

Interchanging the order of integration and summation, we obtain

$$
\begin{aligned}
=e^{-z t} & \sum_{u=0}^{\infty} \sum_{k=0}^{u} \frac{\prod_{j=1}^{p}\left(e_{j}+\nu_{j} k\right)}{\prod_{j=1}^{q}\left(f_{j}+\varepsilon_{j} k\right)} \frac{a^{k}}{k !} \frac{z^{(u-k)}}{(u-k) !} \frac{1}{2 \pi i} \int_{L} \Omega_{p_{i}, q_{i}, \tau_{i}: r}^{m, n}(\xi) y^{-\xi} \\
& \times\left\{\int_{0}^{t} x^{\rho+\varsigma k-\mu \xi-1}(t-x)^{\delta+(\eta-1) k+u-v \xi-1} d x\right\} d \xi,
\end{aligned}
$$


D.L. Suthar, H. Habenom, H. Tadesse - Certain Integrals Involving ...

By replacing $x=t s$, the above equation becomes:

$$
\begin{gathered}
=e^{-z t} t^{\rho+\delta-1} \sum_{u=0}^{\infty} \sum_{k=0}^{u} \frac{\prod_{j=1}^{p}\left(e_{j}+\nu_{j} k\right)}{\prod_{j=1}^{q}\left(f_{j}+\varepsilon_{j} k\right)} \frac{a^{k} t^{u+(\varsigma+\eta-1) k}}{k !} \frac{z^{(u-k)}}{(u-k)} ! \\
\times \frac{1}{2 \pi i} \int_{L} \Omega_{p_{i}, q_{i}, \tau_{i}: r}^{m, n}(\xi) y^{-\xi} t^{-(\mu+v) \xi}\left\{\int_{0}^{1} s^{\rho+\varsigma k-\mu \xi-1}(1-s)^{\delta+(\eta-1) k+u-v \xi-1} d s\right\} d \xi,
\end{gathered}
$$

By using the definition of Beta function

$$
\begin{gathered}
=e^{-z t} t^{\rho+\delta-1} \sum_{u=0}^{\infty} \sum_{k=0}^{u} \frac{\prod_{j=1}^{p}\left(e_{j}+\nu_{j} k\right)}{\prod_{j=1}^{q}\left(f_{j}+\varepsilon_{j} k\right)} \frac{a^{k} t^{u+(\varsigma+\eta-1) k}}{k !} \frac{z^{(u-k)}}{(u-k) !} \\
\times \frac{1}{2 \pi i} \int_{L} \Omega_{p_{i}, q_{i}, \tau_{i}: r}^{m, n}(\xi) \frac{\Gamma(\rho+\varsigma k-\mu \xi) \Gamma(\delta+u+(\eta-1) k-v \xi)}{\Gamma(\rho+\delta+u+(\varsigma+\eta-1) k-(\mu+v) \xi)} y^{-\xi} t^{-(\mu+v) \xi} d \xi
\end{gathered}
$$

Finally, interpreting the contour integral by the virtue of (1) in the right-hand side of the equation (13), we arrive at the desired result (11).

\section{Second Integral}

$$
\begin{aligned}
& I_{2} \equiv \int_{0}^{t} x^{\rho-1}(t-x)^{\delta-1} e^{-x z}{ }_{p} \psi_{q}\left(a x^{\varsigma}(t-x)^{\eta}\right) \\
& \times \aleph_{p_{i}, q_{i}, \tau_{i}: r}^{m, n}\left[y x^{-\mu}(t-x)^{-v} \mid \begin{array}{l}
\left(a_{j}, \alpha_{j}\right)_{1, n}, \ldots,\left[\tau_{i}\left(a_{j i}, \alpha_{j i}\right)\right]_{n+1, p_{i} ; r} \\
\left(b_{j}, \beta_{j}\right)_{1, m}, \ldots,\left[\tau_{i}\left(b_{j i}, \beta_{j i}\right)\right]_{m+1, q_{i} ; r}
\end{array}\right] d x \\
& =e^{-z t} t^{\rho+\delta-1} \sum_{u=0}^{\infty} \sum_{k=0}^{u} \Lambda(k) \frac{z^{(u-k)}}{(u-k) !} t^{u+(\varsigma+\eta-1) k} \\
& \times \aleph_{p_{i}+1, q_{i}+2, \tau_{i}: r}^{m+2, n}\left[\begin{array}{l|l}
y t^{-(\mu+v)} \mid \begin{array}{c}
\left(a_{j}, \alpha_{j}\right)_{1, n}, \ldots,\left[\tau_{i}\left(a_{j i}, \alpha_{j i}\right)\right]_{n+1, p_{i} ; r},(\rho+\delta+u+(\varsigma+\eta-1) k, \mu+v), \\
(\rho+\varsigma k, \mu),(u+\delta-(\eta-1) k, v),\left(b_{j}, \beta_{j}\right)_{1, m}, \ldots,\left[\tau_{i}\left(b_{j i}, \beta_{j i}\right)\right]_{m+1, q_{i} ; r}
\end{array}
\end{array}\right] .
\end{aligned}
$$

provided

$$
\Re(\rho)-\mu \min _{1 \leq j \leq n}\left[\Re\left(\left(a_{j}-1\right) / \alpha_{j}\right)\right]>0, \Re(\delta)-v \min _{1 \leq j \leq n}\left[\Re\left(\left(a_{j}-1\right) / \alpha_{j}\right)\right]>0,
$$

along with the conditions (i) to (iii) given with $\mathrm{I}_{1}$ and $\Lambda(k)$ is given by (12).

\section{Third Integral}

$$
I_{3} \equiv \int_{0}^{t} x^{\rho-1}(t-x)^{\delta-1} e^{-x z} \psi_{q}\left(a x^{\varsigma}(t-x)^{\eta}\right)
$$


D.L. Suthar, H. Habenom, H. Tadesse - Certain Integrals Involving ...

$$
\begin{gathered}
\quad \times \aleph_{p_{i}, q_{i}, \tau_{i}: r}^{m, n}\left[y x^{\mu}(t-x)^{-v} \mid \begin{array}{c}
\left(a_{j}, \alpha_{j}\right)_{1, n}, \ldots,\left[\tau_{i}\left(a_{j i}, \alpha_{j i}\right)\right]_{n+1, p_{i} ; r} \\
\left(b_{j}, \beta_{j}\right)_{1, m}, \ldots,\left[\tau_{i}\left(b_{j i}, \beta_{j i}\right)\right]_{m+1, q_{i} ; r}
\end{array}\right] d x \\
=e^{-z t} t^{\rho+\delta-1} \sum_{u=0}^{\infty} \sum_{k=0}^{u} \Lambda(k) \frac{z^{(u-k)}}{(u-k) !} t^{u+(\varsigma+\eta-1) k} \\
\times \aleph_{p_{i}+1, q_{i}+2, \tau_{i}: r}^{m+1, n+1}\left[\left.y t^{(\mu-v)}\right|_{(u+\delta-(\eta-1) k, v),(1-\rho-\delta-u+(\varsigma+\eta-1) k, \mu-v),\left(b_{j}, \beta_{j}\right)_{1, m}, \ldots,\left[\tau_{i}\left(b_{j i}, \beta_{j i}\right)\right]_{m+1, q_{i} ; r}} ^{(1-\rho+\varsigma k, \mu),\left(a_{j}, \alpha_{j}\right)_{1, n}, \ldots,\left[\tau_{i}\left(a_{j i}, \alpha_{j i}\right)\right]_{n+1, p_{i} ; r}}\right] .
\end{gathered}
$$

provided

$$
\Re(\rho)+\mu \min _{1 \leq j \leq m}\left[\Re\left(b_{j} / \beta_{j}\right)\right]>0, \Re(\delta)-v \min _{1 \leq j \leq n}\left[\Re\left(\left(a_{j}-1\right) / \alpha_{j}\right)\right]>0,
$$

along with the conditions (i) to (iii) given with $\mathrm{I}_{1}$ and $\Lambda(k)$ is given by (12).

\section{Fourth Integral}

$$
\begin{gathered}
I_{4} \equiv \int_{0}^{t} x^{\rho-1}(t-x)^{\delta-1} e^{-x z}{ }_{p} \psi_{q}\left(a x^{\varsigma}(t-x)^{\eta}\right) \\
\times \aleph_{p_{i}, q_{i}, \tau_{i}: r}^{m, n}\left[y x^{-\mu}(t-x)^{v} \mid \begin{array}{c}
\left(a_{j}, \alpha_{j}\right)_{1, n}, \ldots,\left[\tau_{i}\left(a_{j i}, \alpha_{j i}\right)\right]_{n+1, p_{i} ; r} \\
\left(b_{j}, \beta_{j}\right)_{1, m}, \ldots,\left[\tau_{i}\left(b_{j i}, \beta_{j i}\right)\right]_{m+1, q_{i} ; r}
\end{array}\right] d x \\
=e^{-z t} t^{\rho+\delta-1} \sum_{u=0}^{\infty} \sum_{k=0}^{u} \Lambda(k) \frac{z^{(u-k)}}{(u-k) !} t^{u+(\varsigma+\eta-1) k} \\
\times \aleph_{p_{i}+1, q_{i}+2, \tau_{i}: r}^{m+1, n+1}\left[y t^{(-\mu+v)} \mid \begin{array}{c}
(1-u-\delta-(\eta-1) k, v),\left(a_{j}, \alpha_{j}\right)_{1, n}, \ldots,\left[\tau_{i}\left(a_{j i}, \alpha_{j i}\right)\right]_{n+1, p_{i} ; r} \\
(\rho+\varsigma k, \mu),(1-\rho-\delta-u-(\varsigma+\eta-1) k, v-\mu),\left(b_{j}, \beta_{j}\right)_{1, m}, \ldots,\left[\tau_{i}\left(b_{j i}, \beta_{j i}\right)\right]_{m+1, q_{i} ; r}
\end{array}\right] .
\end{gathered}
$$

provided

$$
\Re(\rho)-\mu \min _{1 \leq j \leq n}\left[\Re\left(\left(a_{j}-1\right) / \alpha_{j}\right)\right]>0, \quad \Re(\delta)+v \min _{1 \leq j \leq m}\left[\Re\left(b_{j} / \beta_{j}\right)\right]>0,
$$

along with the conditions (i) to (iii) given with $\mathrm{I}_{1}$ and $\Lambda(k)$ is given by (12).

\section{Special Cases}

On taking $\nu_{j}=\varepsilon_{j}=1$ in equation (11), (14) (15), and (16), we get the following four corollaries respectively, involving generalized hypergeometric function. 


\section{Corollary 1.}

$$
\begin{gathered}
C_{1} \equiv \int_{0}^{t} x^{\rho-1}(t-x)^{\delta-1} e^{-x z}{ }_{p} F_{q}\left(a x^{\varsigma}(t-x)^{\eta}\right) \\
\times \aleph_{p_{i}, q_{i}, \tau_{i}: r}^{m, n}\left[y x^{\mu}(t-x)^{v} \mid \begin{array}{c}
\left(a_{j}, \alpha_{j}\right)_{1, n}, \ldots,\left[\tau_{i}\left(a_{j i}, \alpha_{j i}\right)\right]_{n+1, p_{i} ; r} \\
\left(b_{j}, \beta_{j}\right)_{1, m}, \ldots,\left[\tau_{i}\left(b_{j i}, \beta_{j i}\right)\right]_{m+1, q_{i} ; r}
\end{array}\right] d x \\
=e^{-z t} t^{\rho+\delta-1} \sum_{u=0}^{\infty} \sum_{k=0}^{u} \Theta(k) \frac{z^{(u-k)}}{(u-k) !} t^{u+(\varsigma+\eta-1) k} \\
\times \aleph_{p_{i}+2, q_{i}+1, \tau_{i}: r}^{m, n+2}\left[y t^{\left.(\mu+v) \mid \begin{array}{c}
(1-\rho-\varsigma k, \mu),(1-\delta-(\eta-1) k-u, v),\left(a_{j}, \alpha_{j}\right)_{1, n}, \ldots,\left[\tau_{i}\left(a_{j i}, \alpha_{j i}\right)\right]_{n+1, p_{i} ; r} \\
(1-\rho-\delta-u-(\varsigma+\eta-1) k, \mu+v),\left(b_{j}, \beta_{j}\right)_{1, m}, \ldots,\left[\tau_{i}\left(b_{j i}, \beta_{j i}\right)\right]_{m+1, q_{i} ; r}
\end{array}\right] .}\right.
\end{gathered}
$$

where

$$
\Theta(k)=\frac{\prod_{j=1}^{p}\left(e_{j}+k\right) a^{k}}{\prod_{j=1}^{q}\left(f_{j}+k\right) k !} .
$$

provided that the conditions easily obtainable from those mentioned with (11) are satisfied.

\section{Corollary 2.}

$$
\begin{aligned}
& C_{2} \equiv \int_{0}^{t} x^{\rho-1}(t-x)^{\delta-1} e^{-x z}{ }_{p} F_{q}\left(a x^{\varsigma}(t-x)^{\eta}\right) \\
& \times \aleph_{p_{i}, q_{i}, \tau_{i}: r}^{m, n}\left[y x^{-\mu}(t-x)^{-v} \mid \begin{array}{l}
\left(a_{j}, \alpha_{j}\right)_{1, n}, \ldots,\left[\tau_{i}\left(a_{j i}, \alpha_{j i}\right)\right]_{n+1, p_{i} ; r} \\
\left(b_{j}, \beta_{j}\right)_{1, m}, \ldots,\left[\tau_{i}\left(b_{j i}, \beta_{j i}\right)\right]_{m+1, q_{i} ; r}
\end{array}\right] d x \\
& =e^{-z t} t^{\rho+\delta-1} \sum_{u=0}^{\infty} \sum_{k=0}^{u} \Theta(k) \frac{z^{(u-k)}}{(u-k) !} t^{u+(\varsigma+\eta-1) k} \\
& \times \aleph_{p_{i}+1, q_{i}+2, \tau_{i}: r}^{m+2, n}\left[\begin{array}{l|l}
y t^{-(\mu+v)} \mid \begin{array}{c}
\left(a_{j}, \alpha_{j}\right)_{1, n}, \ldots,\left[\tau_{i}\left(a_{j i}, \alpha_{j i}\right)\right]_{n+1, p_{i} ; r},(\rho+\delta+u+(\varsigma+\eta-1) k, \mu+v), \\
(\rho+\varsigma k, \mu),(u+\delta-(\eta-1) k, v),\left(b_{j}, \beta_{j}\right)_{1, m}, \ldots,\left[\tau_{i}\left(b_{j i}, \beta_{j i}\right)\right]_{m+1, q_{i} ; r}
\end{array}
\end{array}\right] .
\end{aligned}
$$

\section{Corollary 3.}

$$
\begin{gathered}
C_{3} \equiv \int_{0}^{t} x^{\rho-1}(t-x)^{\delta-1} e^{-x z}{ }_{p} F_{q}\left(a x^{\varsigma}(t-x)^{\eta}\right) \\
\times \aleph_{p_{i}, q_{i}, \tau_{i}: r}^{m, n}\left[y x^{\mu}(t-x)^{-v} \mid \begin{array}{l}
\left(a_{j}, \alpha_{j}\right)_{1, n}, \ldots,\left[\tau_{i}\left(a_{j i}, \alpha_{j i}\right)\right]_{n+1, p_{i} ; r} \\
\left(b_{j}, \beta_{j}\right)_{1, m}, \ldots,\left[\tau_{i}\left(b_{j i}, \beta_{j i}\right)\right]_{m+1, q_{i} ; r}
\end{array}\right] d x
\end{gathered}
$$


D.L. Suthar, H. Habenom, H. Tadesse - Certain Integrals Involving ...

$$
\begin{aligned}
& =e^{-z t} t^{\rho+\delta-1} \sum_{u=0}^{\infty} \sum_{k=0}^{u} \Theta(k) \frac{z^{(u-k)}}{(u-k) !} t^{u+(\varsigma+\eta-1) k} \\
& \times \aleph_{p_{i}+1, q_{i}+2, \tau_{i}: r}^{m+1, n+1}\left[y t^{(\mu-v)} \mid \begin{array}{c}
(1-\rho+\varsigma k, \mu),\left(a_{j}, \alpha_{j}\right)_{1, n}, \ldots,\left[\tau_{i}\left(a_{j i}, \alpha_{j i}\right)\right]_{n+1, p_{i} ; r} \\
(u+\delta-(\eta-1) k, v),(1-\rho-\delta-u+(\varsigma+\eta-1) k, \mu-v),\left(b_{j}, \beta_{j}\right)_{1, m}, \ldots,\left[\tau_{i}\left(b_{j i}, \beta_{j i}\right)\right]_{m+1, q_{i} ; r}
\end{array}\right] .
\end{aligned}
$$

\section{Corollary 4.}

$$
\begin{gathered}
C_{4} \equiv \int_{0}^{t} x^{\rho-1}(t-x)^{\delta-1} e^{-x z}{ }_{p} F_{q}\left(a x^{\varsigma}(t-x)^{\eta}\right) \\
\times \aleph_{p_{i}, q_{i}, \tau_{i}: r}^{m, n}\left[y x^{-\mu}(t-x)^{v} \mid \begin{array}{c}
\left(a_{j}, \alpha_{j}\right)_{1, n}, \ldots,\left[\tau_{i}\left(a_{j i}, \alpha_{j i}\right)\right]_{n+1, p_{i} ; r} \\
\left(b_{j}, \beta_{j}\right)_{1, m}, \ldots,\left[\tau_{i}\left(b_{j i}, \beta_{j i}\right)\right]_{m+1, q_{i} ; r}
\end{array}\right] d x \\
=e^{-z t} t^{\rho+\delta-1} \sum_{u=0}^{\infty} \sum_{k=0}^{u} \Theta(k) \frac{z^{(u-k)}}{(u-k) !} t^{u+(\varsigma+\eta-1) k} \\
\times \aleph_{p_{i}+1, q_{i}+2, \tau_{i}: r}^{m+1, n+1}\left[y t^{(-\mu+v)} \mid \begin{array}{c}
(1-u-\delta-(\eta-1) k, v),\left(a_{j}, \alpha_{j}\right)_{1, n}, \ldots,\left[\tau_{i}\left(a_{j i}, \alpha_{j i}\right)\right]_{n+1, p_{i} ; r} \\
(\rho+\varsigma k, \mu),(1-\rho-\delta-u-(\varsigma+\eta-1) k, v-\mu),\left(b_{j}, \beta_{j}\right)_{1, m}, \ldots,\left[\tau_{i}\left(b_{j i}, \beta_{j i}\right)\right]_{m+1, q_{i} ; r}
\end{array}\right] .
\end{gathered}
$$

Remark 3. Using (7) in (15), (16), and (21), make some suitable adjustment of the parameters, we arrive at the known result given by Bhargava et al. [2].

\section{Conclusion}

The Aleph-function, expressed in this paper, is relatively basic in nature. Therefore, on some suitable adjustment of the parameters on function, we may obtain various other special functions such as I-function, Fox's H-function, Meijer's G-function, etc. as its special cases, and therefore, various unified integral presentations can be obtained as special cases of our results. Certain special cases of integrals involving generalized hypergeometric function have been investigated in the literature by some of authors $([1,5,11,12])$ with different arguments. In this sequel, one can obtain integral representation of more generalized special function, which has much application in physics and engineering Science.

\section{REFERENCES}

[1] L.K. Arora, U.K. Saha, Integrals involving Hypergeometric function and $H$ function, J. Indian Acad. Math., 32(1), (2010), 243-249. 
D.L. Suthar, H. Habenom, H. Tadesse - Certain Integrals Involving ...

[2] A. Bhargava, A. Srivastava, R. Mukherjee, Some integrals involving I-function and Wright's generalized Hypergeometric function, Casp. J. of App. Math., Ecology and Economics, 3(1), (2015), 3-11.

[3] A.M. Mathai, R.K. Saxena, H.J. Haubold, The H-Function: Theory and Applications, Springer, New York, (2010).

[4] E.D. Rainville, Special Functions, Chelsea Publishing Co., Bronx, New York, (1971).

[5] U.K. Saha, L.K. Arora, B.K. Dutta, Integrals Involving I-function, Gen. Math. Notes, 6(1),(2011), 1-14.

[6] V.P. Saxena, Formal solution of certain new pair of dual integral equations involving H-functions, Proc. Nat. Acad. Sci. India Sect. A 51, (1982), 366-375.

[7] R.K. Saxena, T.K. Pogány, Mathieu-type series for the §-function occurring in Fokker-Planck equation, Eur. J. Pure Appl. Math., 3(6), (2010), 980-988.

[8] R.K. Saxena, T.K. Pogány, On fractional integration formulae for Aleph functions, Appl. Math. Comput., 218, (2011), 985-990.

[9] N. S̈udland, B. Baumann, T.F. Nannenmacher, Open problem: Who knows about the Aleph-function?, Appl. Anal., 1(4), (1998), 401-402.

[10] N. S̈udland, B. Baumann, T.F. Nannenmacher, Fractional driftless FokkerPlanck equation with power law diffusion coefficients, in V.G. Gangha, E.W. Mayr, W.G. Vorozhtsov (Eds.), Computer Algebra in Scientific Computing (CASC Konstanz 2001), Springer, Berlin, (2001), 513-525.

[11] D.L.Suthar, S. Agarwal, Marichev-Saigo-Maeda fractional integration operator associated with Srivastava's polynomial and Gauss hypergeometric functions, Konuralp J. Math., 5(1),(2016), 145-160.

[12] D.L.Suthar, G.V. Reddy, Biniyam Shimelis, On certain unified fractional integrals pertaining to Product of Srivastava's polynomials and Aleph-function, International Journal of Mathematics Trends and Technology, 47(1),(2017), 66-73.

[13] E.M. Wright, The asymptotic expansion of the generalized hypergeometric function, Proc. London Math. Soc., 46, (1946), 389-408.

D.L. Suthar

Department of Mathematics,

Wollo University,

P.O. Box: 1145, South Wollo, Dessie, Ethiopia

email: dlsuthar@gmail.com

Haile Habenom

Department of Mathematics, 
D.L. Suthar, H. Habenom, H. Tadesse - Certain Integrals Involving ...

Wollo University,

P.O. Box: 1145, South Wollo, Dessie, Ethiopia

email: hhabenom01@gmail.com

Hagos Tadesse

Department of Mathematics,

Wollo University,

P.O. Box: 1145, South Wollo, Dessie, Ethiopia

email: Hagos.tadesse2@gmail.com 